NASA Technical Memorandum 103162

\title{
Two-Dimensional Surface Strain Measurement Based on a Variation of Yamaguchi's Laser-Speckle Strain Gauge
}

John P. Barranger

Lewis Research Center

Cleveland, Ohio

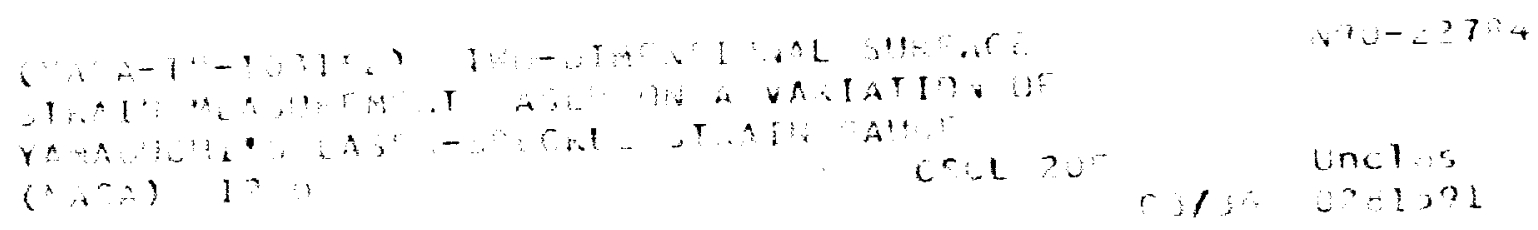

Prepared for the

Conference on Optical Testing and Metrology III sponsored by the Society of Photo-Optical Instrumentation Engineers San Diego, California, July 8-13, 1990 



\title{
Two-Dimensional Surface Strain Measurement Based on a Variation of Yamaguchi's Laser-Speckle Strain Gauge
}

John P. Barranger

NASA Lewis Research Center

Cleveland, Ohio 44135

\begin{abstract}
A novel optical method of measuring two-dimensional surface strain is proposed. Two linear strains along orthogonal axes and the shear strain between those axes is determined by a variation of Yamaguchi's laser-speckle strain gage technique. It offers the advantages of shorter data acquisition times, less stringent alignment requirements, and reduced decorrelation effects when compared to a previously implemented optical strain rosette technique. The method automatically cancels the translational and rotational components of rigid body motion while simplifying the optical system and improving the speed of response.
\end{abstract}

\section{INTRODUCTION}

Elevated temperature tension tests are required to assess the performance of materials intended for use in hostile environments. Conventional wire strain gauges face severe limitations of accuracy, life, and reliability. Non-contact optical techniques can overcome many of the disadvantages of conventional gauges for high temperature strain measurements.

An automatic method of measuring linear surface strain has been described by Yamaguchi $[1,2]$. Two sequentially applied thin laser beams are incident on an optically rough measurement point. The resulting speckle patterns are recorded by a linear detector array that is aligned parallel to the plane of incidence. After straining, another pair of speckle patterns is recorded. These patterns have been displaced relative to the original "reference" patterns. A cross-correlation of the reference and the shifted patterns gives the displacement of each pattern. The difference between speckle displacements is proportional 
to the linear surface strain parallel to the plane of incidence. The optical geometry and use of symmetrically incident beams allow automatic cancellation of any pattern displacements along the plane of incidence due to rigid body motion. The magnitude of cancellation is limited by decorrelation effects [2].

The technique was extended to measure two-dimensional surface strains at a point. Linear strains were determined at three angular orientations by rotating both the plane of incidence and the linear array. Strain rosette equations were applied to calculate the magnitude and direction of the principal strains. The implementation suffered from long data acquisition times, stringent alignment requirements, and decorrelation caused by pattern displacements perpendicular to the direction of the linear array.

An alternative method of determining two-dimensional surface strain is to measure two linear strains along orthogonal axes and the shear strain between those axes. In this paper, a variation of Yamaguchi's technique is described that yields shear surface strain while maintaining the advantages of the original form. An area detector array is used to measure speckle pattern displacements perpendicular as well as parallel to the plane of incidence. The summation of the difference between the perpendicular speckle displacements for each axis results in an expression that is proportional to the shear strain and independent of the translational and rotational components of rigid body motion.

\section{OPTICAL STRAIN ROSETTE SYSTEM WITH LINEAR DETECTOR ARRAY}

Two-dimensional surface strains at a point were measured by extending Yamaguchi's technique [3]. The use of symmetrical beams to cancel rigid body motion, the optical strain rosette implementation, and other system features are described in this section.

\subsection{Speckle Displacement Relations}

The optical geometry of the system is shown in figure 1 . The points $S$ mark the center of curvature $L_{\mathrm{S}}$ of the incident laser wavefronts. Object deformation $a(x, y)$ of the diffuse surface causes speckle displacement $A\left(A_{X}, A_{Y}\right)$ at the observation plane a distance $L_{\mathrm{O}}$ away from the object plane. A laser positioning lens (not shown) provides a nearly flat wavefront at the object plane causing $L_{\mathrm{s}}$ to be much larger than $L_{\mathrm{O}}$. A far-field diffraction pattern is formed at the observation plane on the assumption that $L_{\mathrm{O}}$ is much larger than the spot size of the incident beam. 
The speckle displacements are given by (equations (1), [1])

$$
\begin{aligned}
& A_{X}=a_{x}-L_{0}\left[\varepsilon_{x x \mathrm{~s} x}+\varepsilon_{x y \mathrm{~s} y}-\Omega_{y}\left(l_{\mathrm{s} z}+1\right)+\Omega_{z \mathrm{~s} y}\right] \\
& A_{Y}=a_{y}-L_{0}\left[\varepsilon_{y y s} l_{\mathrm{s} y}+\varepsilon_{x y \mathrm{~s} x} l+\Omega_{x}\left(l_{\mathrm{s} z}+1\right)-\Omega_{z \mathrm{~s} x} l^{l}\right.
\end{aligned}
$$

where $a_{T}\left(a_{x}, a_{y}, a_{z}\right)$ and $\Omega\left(\Omega_{x}, \Omega_{y}, \Omega_{z}\right)$ are the translation vector and rotation vector, respectively. The components of $a_{T}$ and $\Omega$ are the rotational and translational components of rigid body motion, respectively. The unit vector representing the direction of the center $\mathrm{S}$ is denoted by $l_{\mathrm{s}}\left(l_{\mathrm{s} z}, l_{\mathrm{s} y} l_{\mathrm{s} z}\right)$. The linear $x$ and $y$ components of the strain tensor are designated $\varepsilon_{x x}$ and $\varepsilon_{y y^{\prime}}$ respectively, and $\varepsilon_{x y}$ is the in-plane shear component. For the angle of incidence $\theta_{\mathrm{S}}$ in the $x-z$ plane (Fig. 1$), l_{\mathrm{s}}=\left(\sin \theta_{\mathrm{s}}, 0, \cos \theta_{\mathrm{s}}\right)$, and the $X$ and $Y$ components of $A$, denoted by $A_{X, x z}$ and $A_{Y, x z}$ respectively, are given by

$$
\begin{aligned}
& A_{X, x z}=a_{\mathrm{x}}-L_{\mathrm{o}}\left[\varepsilon_{x x} \sin \theta_{\mathrm{s}}-\Omega_{y}\left(\cos \theta_{\mathrm{s}}+1\right)\right] \\
& A_{Y, x z}=a_{y}-L_{\mathrm{o}}\left[\varepsilon_{x y} \sin \theta_{\mathrm{s}}+\Omega_{x}\left(\cos \theta_{\mathrm{s}}+1\right)-\Omega_{z} \sin \theta_{\mathrm{s}}\right] .
\end{aligned}
$$

The speckle displacement $A_{X, x z}$ is measured by one-dimensional cross-correlation of the reference and the shifted speckle patterns. Because the linear array is is aligned parallel to the $X$ axis, the perpendicular displacement $A_{Y, x z}$ cannot be measured by this method.

To eliminate the dependency on the components of rigid body motion, the difference between the speckle displacements for the angles $\theta_{\mathrm{S}}$ and $-\theta_{\mathrm{S}}$ is calculated. Thus,

$$
\Delta A_{X, x z}=A_{X, x z}\left(\theta_{\mathrm{s}}\right)-A_{X, x z}\left(-\theta_{\mathrm{s}}\right)=-2 L_{0} \varepsilon_{x x} \sin \theta_{\mathrm{s}}
$$

and

$$
\varepsilon_{x x}=-\frac{\Delta A_{X, x z}}{2 L_{\mathrm{o}} \sin \theta_{\mathrm{s}}} .
$$

The measurement of linear strain through equation (6) is independent of the rigid body motion components in equation (3). 


\section{$\underline{2.2 \text { Optical Strain Rosette }}$}

To completely characterize two-dimensional surface strain the magnitude and direction of the principal strains must be determined. The required quantities can be obtained from a three-element strain gauge rosette bonded to the surface. The optical analog is the measurement of linear strain at three angular orientations (Fig. 2). This was implemented by rotating both the plane of incidence and the linear array [3]. The principal strains were found from

$$
\begin{aligned}
& \varepsilon_{1}=\frac{1}{2}\left[\varepsilon_{a}+\varepsilon_{c}+\sqrt{\left(\varepsilon_{a}-\varepsilon_{c}\right)^{2}+\left(2 \varepsilon_{b}-\varepsilon_{a}-\varepsilon_{c}\right)^{2}}\right] \\
& \varepsilon_{2}=\frac{1}{2}\left[\varepsilon_{a}+\varepsilon_{c}-\sqrt{\left(\varepsilon_{a}-\varepsilon_{c}\right)^{2}+\left(2 \varepsilon_{b}-\varepsilon_{a}-\varepsilon_{c}\right)^{2}}\right] \\
& \tan 2 \phi=-\frac{\varepsilon_{a}-\varepsilon_{c}}{2 \varepsilon_{b}-\varepsilon_{a}-\varepsilon_{c}}
\end{aligned}
$$

where the two solutions of equation (9) differ by $90^{\circ}$ [4].

\subsection{Optical System Configuration}

The schematic of the optical system is given in figure 3 . The beam of an argon ion laser is switched by an acousto-optic modulator (AOM). Positioning lenses focus the beam to provide a nearly flat wavefront at the specimen surface. Polarization sensitive optics are used to provide the symmetrically incident beams. A polarization beam-splitting cube reflects the beam to the $\theta_{\mathrm{S}}$ side or transmits it to the $-\theta_{\mathrm{S}}$ side, depending on the orientation of the polarization vector. The linear array in the line camera consists of 2048 photodiodes on $15 \mu \mathrm{m}$ centers with a photodiode height $\mathrm{H}$ (Fig. 1) of $0.3 \mathrm{~mm}$. The linear array is one meter away from the specimen surface.

Angular orientation of the plane of incidence and the linear array is achieved by mounting the beam steering optics and the line scan camera on a goniometer cradle. The orientation in figure 2 is dictated by the goniometer which is free to rotate $\pm 45^{\circ}$ about its center point. 
Two quarter-wave $(\lambda / 4)$ retardation plates are used to provide rotational invariance for the polarization sensitive beam steering optics. The first waveplate is stationary and converts the beam from linear to circular polarization; the second waveplate, on the rotating assembly, converts the beam back to linear polarization.

\subsection{Experimental Results}

Static two-dimensional strain measurements were demonstrated using the optical rosette system. Stable speckle patterns were observed for an oxidized superalloy specimen at temperatures up to $750{ }^{\circ} \mathrm{C}$. The total time required to rotate the goniometer to the three orientations and record the six speckle patterns was 10 seconds. This data acquisition time is too long for dynamic applications such as standard elevated temperature tension tests.

The ability to measure the principal strains from the three linear strains depended to a great extent on the performance of the goniometer. The alignment, positioning, and repeatability requirements were all very stringent. Measurement error could have been introduced if extreme care had not been exercised.

Excessive speckle displacement can lead to decorrelation. When the displacement is so great that the areas of the speckles in the reference pattern move off the array then correlation is lost. Yamaguchi [5] defined the correlation limit of the perpendicular displacement $A_{Y, x z}$ (equation (4)) as the sum of the array height $H$ (Fig. 1) and the mean speckle height. Because of the small $A_{Y, x z}$ limit, the system experienced decorrelation perpendicular to the direction of the array.

Similarly, the correlation limit of $A_{X, x z}$ (equation (3)) is the sum of the array length $\mathrm{L}$ and the mean speckle length. When the correlation limit is large, however, the actual decorrelation depends on many practical factors such as specimen movement, material, and surface topology. This was observed in the direction of the array.

\section{SHEAR STRAIN SYSTEM WITH AREA DETECTOR ARRAY}

The system described in the above section suffered from long data acquisition times, stringent alignment requirements, and decorrelation perpendicular to the direction of the array. To overcome these disadvantages, the goniometer is now replaced by additional beam steering optics and an area array is substituted for the linear array. Extending the three element rosette configuration would require three pairs of beam positions for three angular orientations and six two-dimensional (2-D) cross-correlations. A novel 
implementation is proposed in this section which simplifies the system by eliminating one of the angular orientations and two 2-D cross-correlations.

\subsection{Speckle Displacement Relations}

An alternative to the three element rosette method of determining two-dimensional surface strain is to measure two linear strains along orthogonal axes and the shear strain between those axes. As noted by Yamaguchi [1], the shear strain component $\varepsilon_{x y}$ as well as rigid body components are contained in the perpendicular displacement $A_{Y, x z}$ (equation (4)). Direct implementation of the symmetrical beam position concept, however, does not lead to complete rigid body cancellation. An important summation operation is required before the desired result is achieved.

Figure 4, the optical geometry of the system, is the same as figure 1 except for the replacement of the linear array by the area array and the addition of two source points $\mathrm{S}$. The sides of the rectangular array are aligned parallel to the $X$ and $Y$ axes.

For $\theta_{\mathrm{s}}$ in the $x-z$ plane, the $A_{X, x z}$ and $A_{Y, x z}$ components of $A$ are the same as equations (3) and (4), respectively. Both speckle displacements are measured by 2-D cross-correlation of the reference and the shifted patterns using any appropriate means [6]. To eliminate the dependency of $A_{X, x z}$ and reduce the dependency of $A_{Y, x z}$ on the components of rigid body motion, the difference between the speckle displacements for the angles $\theta_{\mathrm{S}}$ and $-\theta_{\mathrm{S}}$ is calculated. Thus, $\Delta A_{X, x z}$ is the same as equation (5), $\varepsilon_{x x}$ is the same as equation (6), and

$$
\Delta A_{Y, x z}=A_{Y, x z}\left(\theta_{\mathrm{S}}\right)-A_{Y, x z}\left(-\theta_{\mathrm{S}}\right)=-2 L_{0}\left(\varepsilon_{x y} \sin \theta_{\mathrm{S}}-\Omega_{z} \sin \theta_{\mathrm{S}}\right)
$$

Equation (10) retains the shear strain component but $\Omega_{z}$ is not canceled by using the symmetrical beam positions.

Beam steering optics now change the plane of incidence by $90^{\circ}$. For $\theta_{\mathrm{s}}$ in the $y-z$ plane (Fig. 4), $l_{\mathrm{s}}=\left(0, \sin \theta_{\mathrm{S}}, \cos \theta_{\mathrm{s}}\right)$, and the components of $A$ are given by

$$
\begin{aligned}
& A_{X, y z}=a_{x}-L_{0}\left[\varepsilon_{x y} \sin \theta_{\mathrm{s}}-\Omega_{y}\left(\cos \theta_{\mathrm{s}}+1\right)+\Omega_{z} \sin \theta_{\mathrm{s}}\right] \\
& A_{Y, y z}=a_{y}-L_{0}\left[\varepsilon_{y y} \sin \theta_{\mathrm{s}}+\Omega_{x}\left(\cos \theta_{\mathrm{s}}+1\right)\right] .
\end{aligned}
$$

-For this orientation, the perpendicular displacement is $A_{X, y z}$ Taking the difference between the speckle displacements for the angles $\theta_{\mathrm{S}}$ and $-\theta_{\mathrm{S}}$ yields the equations, 


$$
\begin{aligned}
& \Delta A_{X, y z}=A_{X, y z}\left(\theta_{\mathrm{s}}\right)-A_{X, y z}\left(-\theta_{\mathrm{s}}\right)=-2 L_{\mathrm{o}}\left(\varepsilon_{x y} \sin \theta_{\mathrm{s}}+\Omega_{z} \sin \theta_{\mathrm{s}}\right) \\
& \Delta_{Y, y z}=A_{Y, y z}\left(\theta_{\mathrm{s}}\right)-A_{Y, y z}\left(-\theta_{\mathrm{s}}\right)=-2 L_{0} \varepsilon_{y y} \sin \theta_{\mathrm{s}} .
\end{aligned}
$$

Finally, the shear component is completely separated by combining the difference between the perpendicular displacements for each orientation. Summing equations (10) and (13),

$$
\Delta A_{Y, x z}+\Delta A_{X, y z}=-4 L_{\mathrm{o}} \varepsilon_{x y} \sin \theta_{\mathrm{s}}
$$

The linear strain $\varepsilon_{y y}$ and the shear strain $\gamma_{x y}$ are found from

$$
\begin{aligned}
\varepsilon_{y y} & =-\frac{\Delta A_{Y, y z}}{2 L_{0} \sin \theta_{\mathrm{s}}} \\
\gamma_{x y} & =-\frac{\Delta A_{Y, x z}+\Delta A_{X, y z}}{2 L_{0} \sin \theta_{\mathrm{s}}}
\end{aligned}
$$

where $\varepsilon_{x y}$ in equation (15) has been replaced by $\gamma_{x y} / 2$. Thus, equations (6), (16), and (17) represent the desired two linear strains along orthogonal axes and the shear strain between those axes.

The magnitude and direction of the principal strains are found from the equations

$$
\begin{aligned}
& \varepsilon_{1}=\frac{1}{2}\left[\varepsilon_{x x}+\varepsilon_{y y}+\sqrt{\left(\varepsilon_{x x}-\varepsilon_{y y}\right)^{2}+\gamma_{x y}^{2}}\right] \\
& \varepsilon_{2}=\frac{1}{2}\left[\varepsilon_{x x}+\varepsilon_{y y}-\sqrt{\left(\varepsilon_{x x}-\varepsilon_{y y}\right)^{2}+\gamma_{x y}^{2}}\right]
\end{aligned}
$$

$$
\tan 2 \phi=\frac{\gamma_{x y}}{\varepsilon_{x x}-\varepsilon_{y y}}
$$

where the two solutions of equation (20) differ by $90^{\circ}[4]$. 


\subsection{Area Array Results}

A commercial frame transfer charge-coupled device has been selected for the area array of the proposed system. It consists of 739 photosites on $8.5 \mu \mathrm{m}$ centers parallel to the $X$ axis and 484 photosites on $9.875 \mu \mathrm{m}$ centers parallel to the $Y$ axis. Synchronization and timing follow the EIA RS-170 standard operating at a frame rate of $30 \mathrm{~Hz}$.

By steering the laser beams during the transfer gate period (about $30 \mu \mathrm{s}$ ) of vertical blanking, the total time to acquire the four speckle patterns is reduced to $4 / 30$ or 0.13 seconds. This is a 75 fold improvement in the acquisition time over the optical rosette system.

The area array reduces decorrelation caused by excessive speckle displacement. Extending the results of the optical rosette system, the correlation limits still apply except that $\mathrm{L}$ and $\mathrm{H}$ are now the area array length and height, respectively (Fig. 4). The larger value of $\mathrm{H}(4.78 \mathrm{~mm}$ compared to $0.3 \mathrm{~mm})$ greatly extends the correlation limit in the direction parallel to the $Y$ axis. In spite of the smaller value of $\mathrm{L}(6.28 \mathrm{~mm}$ compared to $30.7 \mathrm{~mm}$ ), from a practical point of view the correlation limit in the direction parallel to the $X$ axis suffers only slightly.

\section{CONCLUDING REMARKS}

Compared to the earlier implementation, the use of the area array reduces data acquisition times, eliminates the stringent alignment of the goniometer, and reduces decorrelation effects. Moreover, this method employs two pairs of beam positions rather than three and reduces the number of two-dimensional cross-correlation calculations from six to four. These features simplify the optical system and improve the speed of response.

\subsection{REFERENCES}

1. Yamaguchi, I., A Laser-Speckle Strain Gauge. J. Phys. E. Sci. Instrum., vol. 14, no. 11, pp. 1270-1273, Nov. 1981.

2. Yamaguchi, I., Speckle Displacement and Decorrelation in the Diffraction and Image Fields for Small Object Deformation. Optica Acta, vol. 28, no. 10, pp. 1359-1376, Oct. 1981.

3. Lant, C.T., Two-Dimensional High Temperature Optical Strain Measurement System - Phase II. NASA CR-185116, 1989. 
4. Kobayashi, A.S., Ed., Handbook on Experimental Mechanics, Prentice-Hall, Inc., pp. 113-115, 1987.

5. Yamaguchi, I., Simplified Laser-Speckle Strain Gauge. Optical Engineering, vol. 21, no. 3, p. 437, May/June 1982.

6. Takai, N.; Asakura, T., Vectorial Measurements of Speckle Displacement by the 2-D Electronic Correlation Method. Appl. Opt., vol. 24, no. 5, pp. 660-665, 1 March 1985.

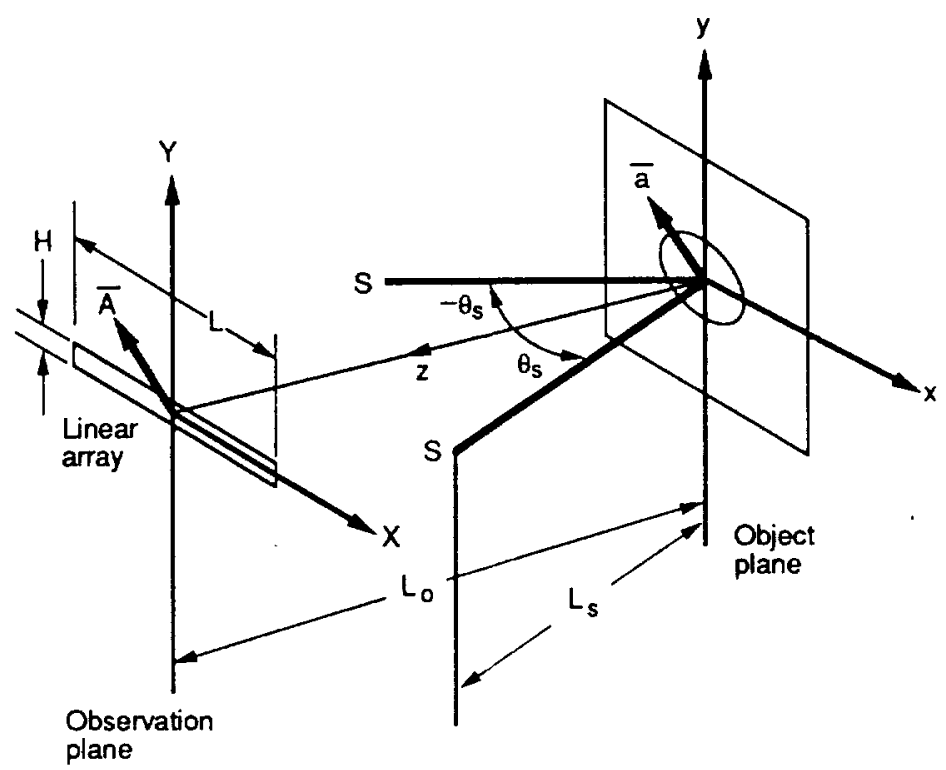

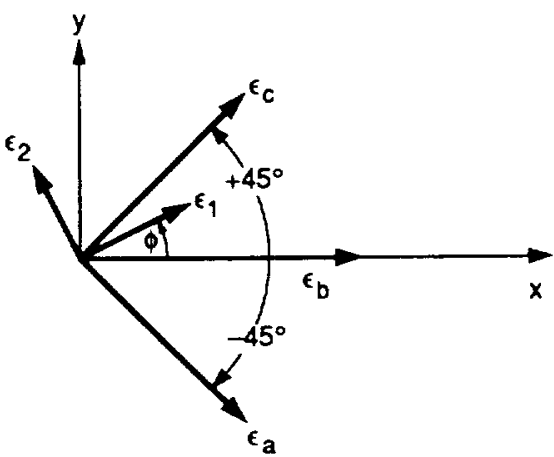

Figure 2. - Angular orientation of optical strain rosette.

Figure 1. - Optical geometry of optical strain rosette system with linear detector array.

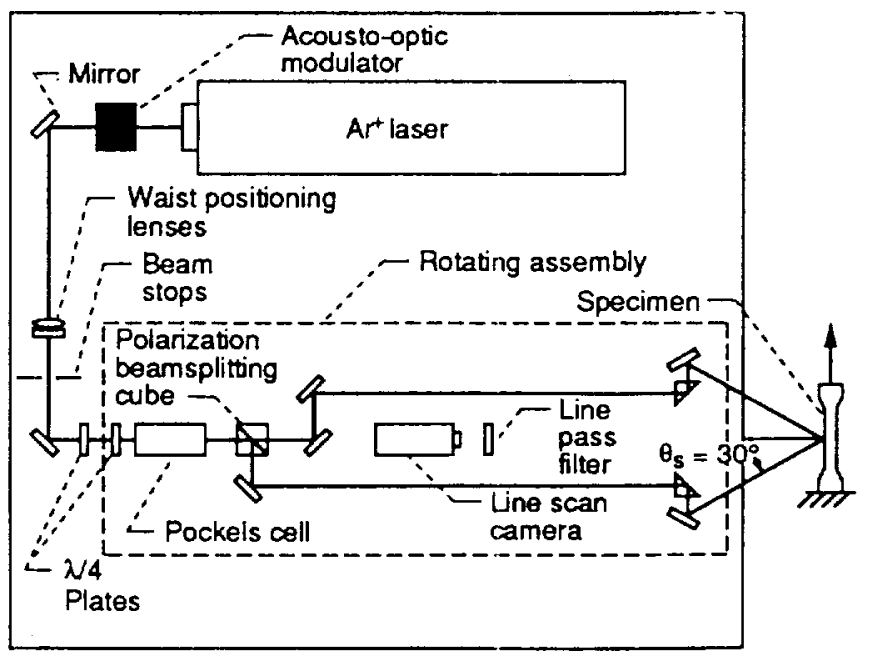

Figure 3. - Optical system configuration. 


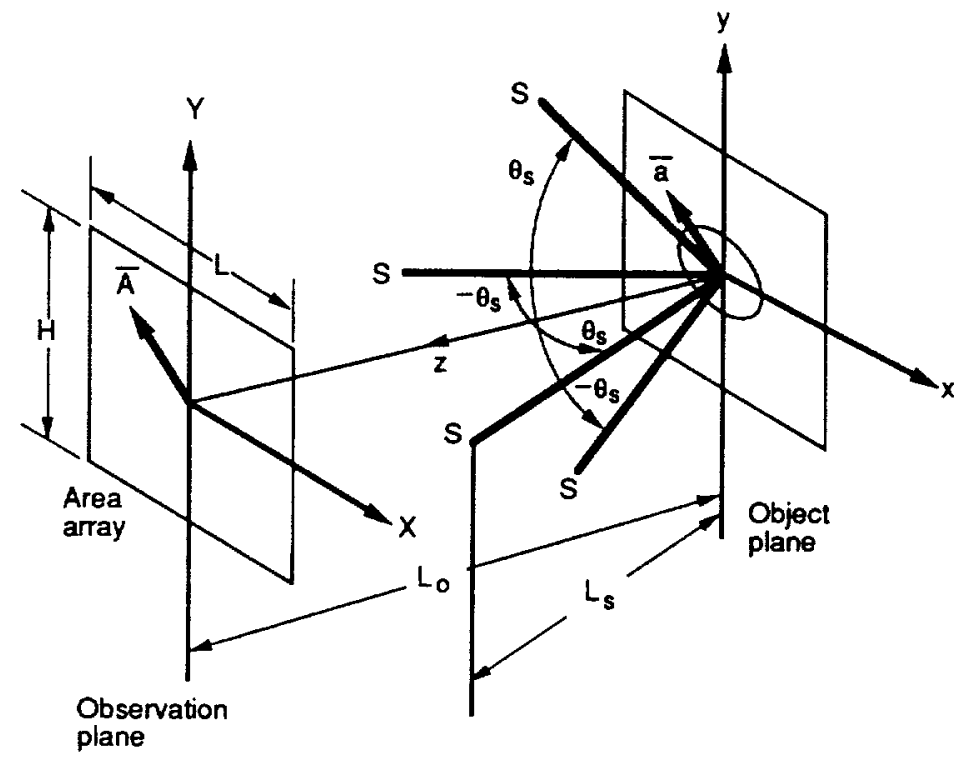

Figure 4. - Optical geometry of shear strain system with area detector array. 


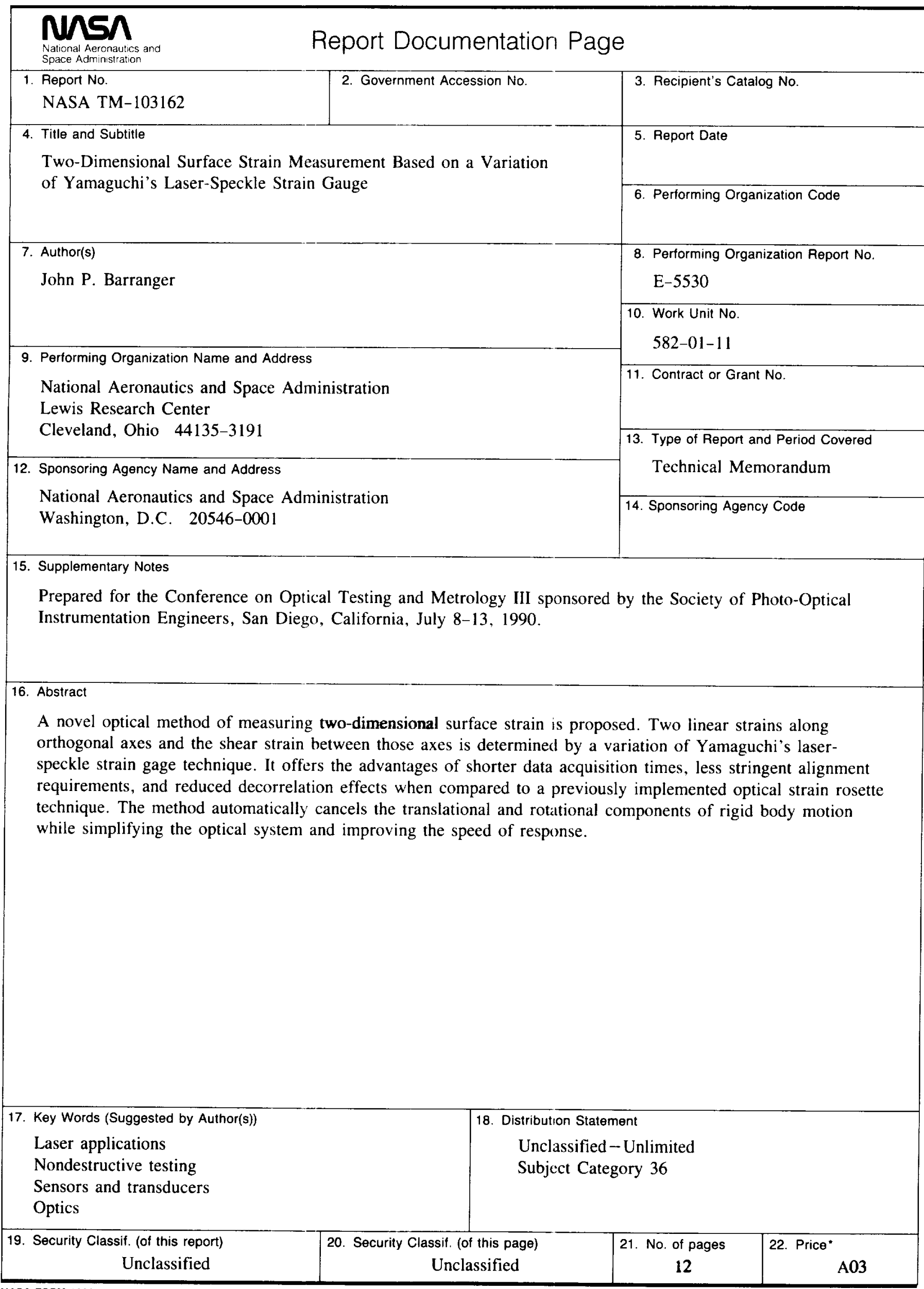


